\title{
High-resolution model of Asteroid 4179 Toutatis
}

\author{
R.S. Hudson, ${ }^{a}$ S.J. Ostro, ${ }^{\mathrm{b}}$ and D.J. Scheeres ${ }^{\mathrm{c}, *}$ \\ ${ }^{a}$ Washington State University Tri-Cities, 2710 University Drive, Richland, WA 99352-1671, USA \\ ${ }^{\mathrm{b}}$ Jet Propulsion Laboratory, California Institute of Technology, Pasadena, CA 91109-8099, USA \\ ${ }^{\mathrm{c}}$ Department of Aerospace Engineering, University of Michigan, 3048 FXB, Ann Arbor, MI 48109-2140, USA
}

Received 22 August 2001; revised 13 September 2002

\begin{abstract}
A model of the shape of Toutatis based on "high-resolution" radar images obtained in 1992 and 1996 consists of 39,996 triangular facets of roughly equal area, defined by the locations of 20,000 vertices. These define the average spatial resolution of the model as approximately 34 m, significantly finer than Hudson and Ostro's (1995, Science 270, 84-86) model (1600 vertices, resolution 84 m). The high-resolution model reveals complex linear features as well as circular crater-like structures down to the resolution limit. There is a dichotomy between the gravitational slope distributions on the asteroid's two lobes. The noncraterlike features may be the manifestation of complex interior configurations involving monolithic fragments with various sizes and shapes, presumably due to collisions in various energy regimes. However, it may be impossible to decipher the interior structure and responsible collisional history of Toutatis and other small objects without extended, intensive, direct examination.
\end{abstract}

(C) 2003 Elsevier Science (USA). All rights reserved.

Keywords: Asteroids; Radar; Photometry; Image processing

\section{Introduction}

Delay-Doppler radar imaging during Toutatis' Dec. 1992 close approach (to within $0.024 \mathrm{AU}$, or 9.4 lunar distances) provided information that remains unprecedented for an Earth-crossing object (Ostro et al., 1995). Hudson and Ostro (1995, hereafter HO95) used a comprehensive physical model to invert the lower-resolution images to estimate the asteroid's detailed shape and inertia tensor, initial conditions for the asteroid's spin and orientation, the radar scattering properties of the surface, and the delay-Doppler trajectory of the center of mass. They found that Toutatis is rotating in a long-axis mode characterized by periods of 5.4 days (rotation about the long axis) and 7.4 days (average for long-axis precession about the angular momentum vector). The asteroid's maximum extents along the principal axes are $(1.92,2.29,4.60) \pm 0.10 \mathrm{~km}$. The dynamically equivalent, equal volume ellipsoid (DEEVE), that is, the ellipsoid that has the same moment ratios and the same volume as the

\footnotetext{
* Corresponding author. Fax: +1-734-763-0578.

E-mail address: scheeres@umich.edu (D.J. Scheeres).
}

asteroid, has dimensions $(1.70,2.03,4.26) \pm 0.08 \mathrm{~km}$. The inertia tensor is indistinguishable from that of a homogeneous body with Toutatis' shape.

Using the low-resolution shape model (Fig. 1), Hudson and Ostro (1998) analyzed optical lightcurves (Spencer et al., 1995) and produced estimates of the object's Hapke parameters and a slight refinement of the spin state. That model also was used by Scheeres et al. (1998) to examine the dynamics of orbits very close to Toutatis and by Scheeres et al. (2000) to explore the possible role of gravitational interactions in the origin of Toutatis' spin state.

Observations of Toutatis during its 1996 approach to within $0.035 \mathrm{AU}$ produced nine consecutive days of delayDoppler and Doppler-only measurements, increasing the radar time base from three weeks to four years and thereby permitting significant refinements in our knowledge of the asteroid's orbit, spin state, shape, and radar-scattering properties, as follows (Ostro et al., 1999). This experiment, which confirmed the accuracy of predictions of the progression of Toutatis' appearance in delay-Doppler images (Hudson and Ostro, 1996), showed that the asteroid's surface properties are strikingly uniform, provided evidence for a 


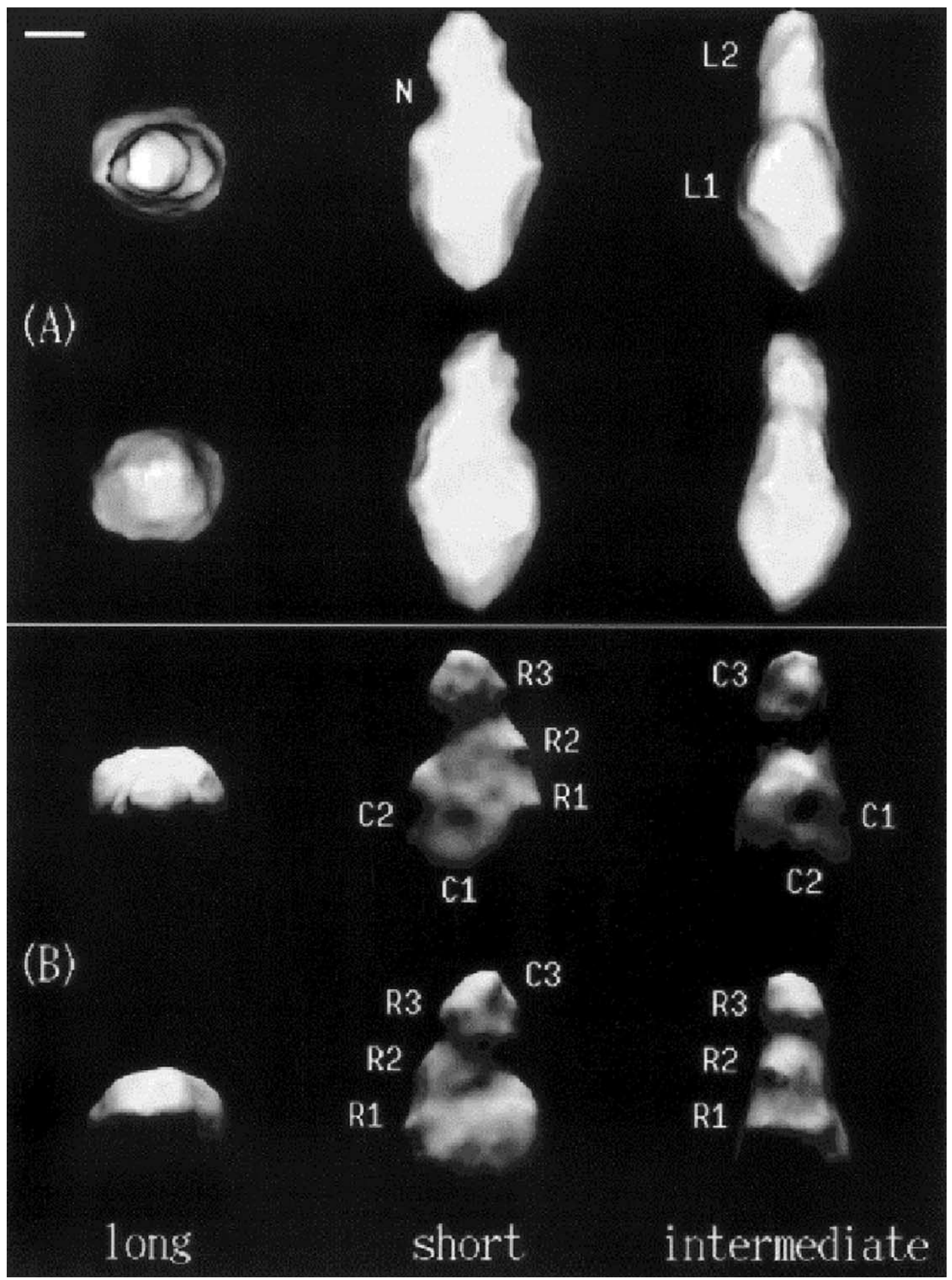

Fig. 1. The low-resolution Toutatis model (Fig. 1 of Hudson and Ostro, 1995). (A) Renderings of the model from both directions on the long, short, and intermediate axes. The bar at the upper left is $1 \mathrm{~km}$ long. Source and observer are collocated. (B) Same as (A), but the source of illumination is moved $75^{\circ}$ toward the top of the page. Lambertian scattering was used in all renderings. Features: L, lobe, N, neck, C, concavity, and R, ridge.

smooth surface component, and are consistent with Toutatis' surface having a smooth component that is at least one-third covered by rocks at least as large as the wavelength. If this S-class object is mineralogically similar to stony-iron meteorites, then the smooth surface component probably is regolith whose porosity resembles that of lunar soil. If the mineralogy is ordinary chondritic, then the smooth surface component probably is solid with not much more than a centimeter of overlying regolith.

The HO95 model used low-resolution images, with delay resolution of $500 \mathrm{~ns}$ and Doppler resolution of $0.1 \mathrm{~Hz}$, that were taken during a short period on each date to validate the 


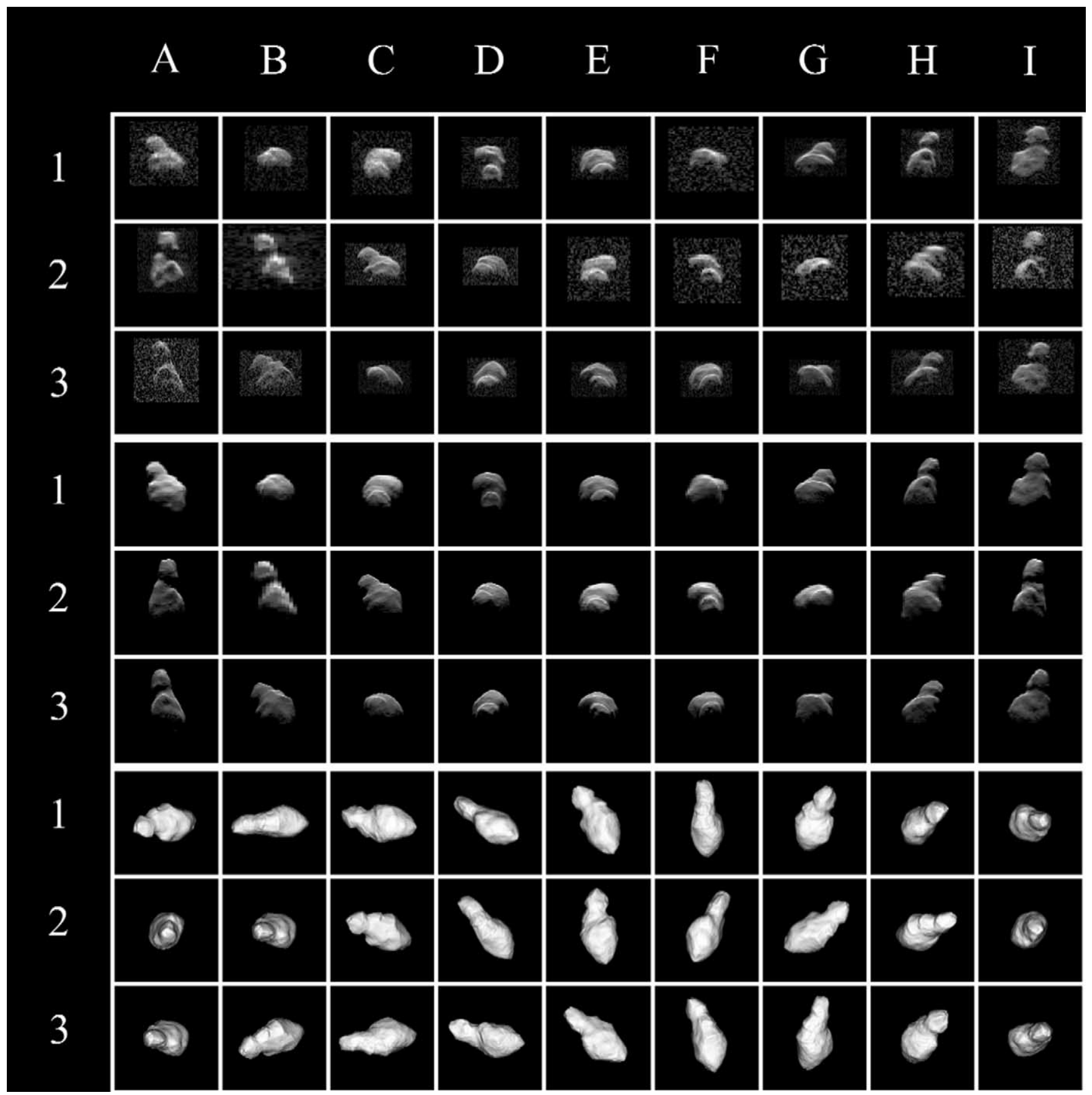

Fig. 2. Top block of three rows shows observed delay-Doppler images. Delay increases from top to bottom and Doppler frequency increases from left to right. The middle three-row block shows model fits to those images, and the bottom three-row block shows corresponding plane-of-sky views of the high-resolution model. All frames are $6 \times 6 \mathrm{~km}$ and are centered on Toutatis' center of mass. Labels correspond to those in Table 1 .

integrity of the radar system. The most informative Toutatis images, obtained on eight dates in 1992 and on nine dates in 1996, have delay resolution of $125 \mathrm{~ns}$ and typical Doppler resolution of $0.033 \mathrm{~Hz}$, that is, with delay $\times$ Doppler resolution 12 times finer than in the data used by HO95. Here we present a shape model that incorporates those 17 highresolution images as well as lower-delay-resolution images from the 10 dates in 1992 on which no high-resolution images were obtained. This new model's intrinsic areal resolution, $(\sim 34 \mathrm{~m})^{2}$, is significantly finer than the HO95 model's areal resolution, $(\sim 84 \mathrm{~m})^{2}$, and is comparable to those of Galileo's highest-resolution images of Dactyl (Veverka et al., 1996) and NEAR Shoemaker's first images of Eros after orbit insertion.

We believe that in this paper we are demonstrating the degree to which groundbased radar can achieve reconstruction of an asteroid's shape with fine fractional resolution over the entire surface. Some other asteroid radar data sets 
Table 1

Images used in the shape reconstruction

\begin{tabular}{|c|c|c|c|c|c|c|c|c|c|}
\hline Label & Date & hh:mm & TX, RCV & File & $N$ & $\begin{array}{l}D_{\mathrm{t}} \\
(\mathrm{ns})\end{array}$ & $\begin{array}{l}D_{\mathrm{f}} \\
(\mathrm{Hz})\end{array}$ & $\mathrm{km} / \mathrm{Hz}$ & $\begin{array}{l}D_{\mathrm{f}} \times D_{\mathrm{t}} \\
(\mathrm{m} \times \mathrm{m})\end{array}$ \\
\hline $1 \mathrm{~A}$ & 1992 Dec 2 & $21: 40$ & 14,14 & 33707 & 7 & 500 & 0.100 & 0.96 & $96 \times 75$ \\
\hline 1B & 1992 Dec 3 & $19: 30$ & 14,14 & 33804 & 14 & 500 & 0.100 & 0.86 & $86 \times 75$ \\
\hline $1 \mathrm{C}$ & $1992 \operatorname{Dec} 4$ & $18: 10$ & 14,14 & 33904 & 14 & 500 & 0.100 & 0.85 & $85 \times 75$ \\
\hline $1 \mathrm{D}$ & 1992 Dec 5 & $18: 50$ & 14,14 & 34006 & 22 & 125 & 0.083 & 0.89 & $74 \times 19$ \\
\hline $1 \mathrm{E}$ & 1992 Dec 6 & $17: 30$ & 14,13 & 34107 & 104 & 125 & 0.083 & 0.95 & $79 \times 19$ \\
\hline $1 \mathrm{~F}$ & 1992 Dec 7 & $17: 20$ & 14,13 & 34204 & 83 & 500 & 0.100 & 1.12 & $112 \times 75$ \\
\hline $1 \mathrm{G}$ & 1992 Dec 8 & $16: 40$ & 14,13 & 34307 & 106 & 125 & 0.033 & 1.39 & $46 \times 19$ \\
\hline $1 \mathrm{H}$ & $1992 \operatorname{Dec} 9$ & $17: 50$ & 14,13 & 34403 & 25 & 125 & 0.033 & 1.78 & $59 \times 19$ \\
\hline $1 \mathrm{I}$ & 1992 Dec 10 & $17: 20$ & 14,13 & 34504 & 72 & 125 & 0.033 & 2.90 & $96 \times 19$ \\
\hline $2 \mathrm{~A}$ & 1992 Dec 11 & $10: 30$ & 14,13 & 34606 & 106 & 125 & 0.008 & 4.8 & $40 \times 19$ \\
\hline $2 \mathrm{~B}$ & 1992 Dec 12 & 09:20 & 14,14 & 34703 & 18 & 500 & 0.100 & 2.13 & $213 \times 75$ \\
\hline $2 \mathrm{C}$ & 1992 Dec 13 & 09:10 & 14,13 & 34805 & 162 & 125 & 0.033 & 1.17 & $39 \times 19$ \\
\hline $2 \mathrm{D}$ & 1992 Dec 14 & 09:00 & 14,13 & 34906 & 99 & 125 & 0.033 & 0.92 & $30 \times 19$ \\
\hline $2 \mathrm{E}$ & 1992 Dec 15 & $07: 50$ & 14,14 & 35002 & 29 & 500 & 0.100 & 0.86 & $86 \times 75$ \\
\hline $2 \mathrm{~F}$ & 1992 Dec 16 & $07: 10$ & 14,14 & 35104 & 18 & 500 & 0.100 & 0.86 & $86 \times 75$ \\
\hline $2 \mathrm{G}$ & 1992 Dec 17 & $06: 50$ & 14,14 & 35204 & 27 & 500 & 0.100 & 0.90 & $86 \times 75$ \\
\hline $2 \mathrm{H}$ & 1992 Dec 18 & $07: 10$ & 14,14 & 35304 & 151 & 500 & 0.100 & 1.15 & $86 \times 75$ \\
\hline $2 \mathrm{I}$ & 1992 Dec 19 & $08: 40$ & $\mathrm{~A}, \mathrm{~A}$ & & 46 & 200 & 0.012 & 5.67 & $70 \times 30$ \\
\hline $3 \mathrm{~A}$ & 1996 Nov 25 & $19: 48$ & 14,13 & 33010 & 11 & 125 & 0.033 & 1.28 & $43 \times 19$ \\
\hline $3 \mathrm{~B}$ & 1996 Nov 26 & $17: 54$ & 14,13 & 33102 & 75 & 125 & 0.033 & 1.05 & $35 \times 19$ \\
\hline $3 C$ & 1996 Nov 27 & $17: 47$ & 14,13 & 33205 & 130 & 125 & 0.033 & 0.905 & $30 \times 19$ \\
\hline $3 \mathrm{D}$ & 1996 Nov 28 & $17: 32$ & 14,13 & 33304 & 161 & 125 & 0.033 & 0.895 & $30 \times 19$ \\
\hline $3 \mathrm{E}$ & 1996 Nov 29 & $15: 35$ & 14,13 & 33402 & 154 & 125 & 0.033 & 0.911 & $30 \times 19$ \\
\hline $3 \mathrm{~F}$ & 1996 Nov 30 & $14: 37$ & 14,13 & 33502 & 160 & 125 & 0.033 & 0.951 & $30 \times 19$ \\
\hline $3 G$ & 1996 Dec 1 & $13: 23$ & 14,13 & 33602 & 144 & 125 & 0.033 & 1.04 & $34 \times 19$ \\
\hline $3 \mathrm{H}$ & 1996 Dec 2 & $13: 09$ & 14,13 & 33702 & 128 & 125 & 0.033 & 1.22 & $40 \times 19$ \\
\hline $3 I$ & 1996 Dec 3 & $12: 08$ & 14,13 & 33803 & 200 & 125 & 0.033 & 1.81 & $60 \times 19$ \\
\hline
\end{tabular}

1992 and 1996 images reported by Ostro et al. (1995 and 1999, respectively) are listed chronologically. UTC midpoints of reception are given for each image. Goldstone $(8510-\mathrm{MHz}, 3.5-\mathrm{cm})$ observations used the 70-m antenna, DSS-14, for transmission. The sixth column (TX, RCV) gives transmit and receive antennas. Some Goldstone observations were bistatic, done by transmitting continuously from DSS-14 and receiving at DSS-13, a 34-m antenna 22 km away. The single Arecibo (2380-MHz, 13-cm) observation (A) was monostatic. Goldstone image designations are the day of year followed by a two-digit index. $N$ is the number of looks summed. The time-delay and Doppler frequency resolutions $D_{\mathrm{t}}$ and $D_{\mathrm{f}}$ that is, the image pixel dimensions, are given along with their length equivalents. The frequency resolution $D_{\mathrm{f}}$ has a length equivalent of $D_{\mathrm{f}}$ times the $\mathrm{km} / \mathrm{Hz}$ conversion factor, determined by the asteroid's spin state.

involve objects as large as Toutatis, or absolute/fractional resolution comparable to the Toutatis images or have yielded 3-D models, but no other Earth-crossing asteroid radar data set achieves global coverage with similarly fine fractional and absolute resolution, or has produced a physical model with unambiguous determination of the spin state (including moment of inertia ratios).

\section{Modeling}

We used the 26 Goldstone and single Arecibo delayDoppler images shown in Fig. 2 and described in Table 1. In each case, the image used has the highest resolution and/or the highest signal-to-noise ratio of any taken on that date. Our initial condition for modeling was the HO95 shape model with a spin state refined by optical data (HO95) and 1996 radar data (Ostro et al., 1999). Our modeling procedure was essentially the same as previously described for the low-resolution model. We iteratively added vertices (typically doubling the number each time) and refit to convergence. We stopped when the number of vertices $(20,000)$ was large enough so that we felt confident that our model was able to accurately represent the data set without introducing spurious surface structure.

Delay-Doppler images involve a highly counterintuitive projection and a possibly many-to-one mapping from the surface to the image. For example, in looking at radar images of asteroids, one often notices shadows that suggest surface relief or a prominent concavity in the shape. But radar images are taken with the transmitter and receiver collocated, that is, at opposition, so the radar cannot "see" any shadows at all. This paradox is an outcome of the fact that radar images are projections along the line of sight rather than in the plane of the sky.

For a rotating rigid body, the delay-Doppler trajectory of any surface point is a unique curve. Therefore, a sequence of images providing adequate orientational coverage can, in principle, be inverted to produce an estimate of the asteroid's shape. Our inversion uses a weighted-least-squares estimator to solve a highly nonlinear system. Whereas many structural features in our model are prominent in images, more subtle features that are not easily seen in individual images can be discerned through the least-squares estima- 

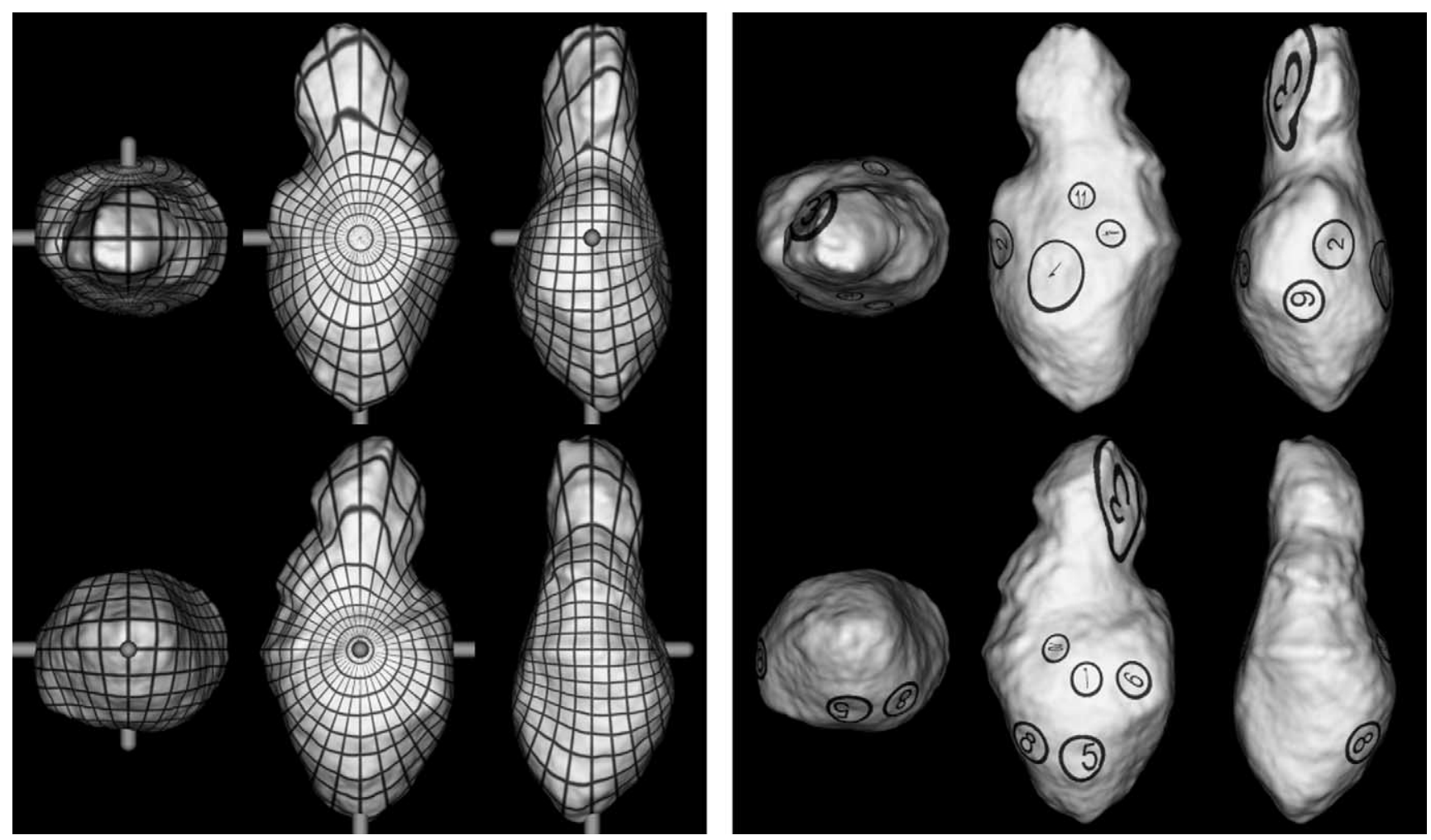

Fig. 3. Toutatis model viewed along its principal axes of inertia as in Fig. 1, which shows the low-resolution model of HO95. In the left frame, cylinders protruding from the model are the positive $x, y, z$ axes. The longitude-latitude grid, drawn at $10^{\circ}$ intervals, uses the $z$ axis as the pole, which would be the case if the asteroid's rotation were damped to its minimum-energy principal-axis state. On the right, we have circled 11 concavity features that are prominent in the delay-Doppler images.

tor, because structural features are identifiable by their multiframe delay-Doppler trajectories. In different words, a feature's signature in any given frame might be marginally significant while its collective effect through multiple frames would be significant, albeit discernible only through the "eyes" of the least-squares estimator. This situation is common whenever observations are related nonlinearly to model parameters (e.g., Bard, 1974).

We went to some length to balance trying to squeeze every last drop of information out of the images against misinterpreting noise in the data as surface structure. A primary tool for doing this involved inclusion of a "relief penalty" term in the objective function that we minimized to identify the least-squares solution. This term suppresses both positive and negative relief. We have drawn on our experience with both numerical simulations and laboratory experiments with a laser-radar/model-asteroid system (Andrews et al., 1995), in an effort to be conservative but not overly so. A consequence of this effort is that it is much more likely that Toutatis has structure not evident in our model than that Toutatis does not have structure that is evident in our model: we have chosen to prefer understating relief rather than to risk overstating it.

Fig. 2 also shows the delay-Doppler fits produced by the model and the corresponding plane-of-sky views. Fig. 3 shows views of the model along principal-axis directions, in the same format as for the low-resolution model in Fig. 1, with 11 of the most prominent concavity features on the surface identified. We have rendered the model using zerophase illumination with a Lambertian scattering law. We caution readers that our figures show a shape model, not photographs of the asteroid. The nature of the delay-Doppler projection is intrinsically nonintuitive and very often counterintuitive. For readers used to interpreting spacecraft images of asteroids, it may be useful to imagine trying to interpret only shape models based on those images without access to the images themselves.

\section{Results}

In its current spin state (see complete description by Scheeres et al., 1998), the object's instantaneous spin vector and equator are periodic functions. Complete damping to uniform rotation about the maximum-inertia axis (its shortest one) would result in a 7.35-day spin, and the equatorial plane would be that containing the other principal axes. In Fig. 3, the contours of latitude and longitude are based on the future pole and equator; these could be used as a cartographic coordinate system. However, our shape model uses $x, y, z$ Cartesian coordinates, and we will use these as the basis for our discussion. 
a

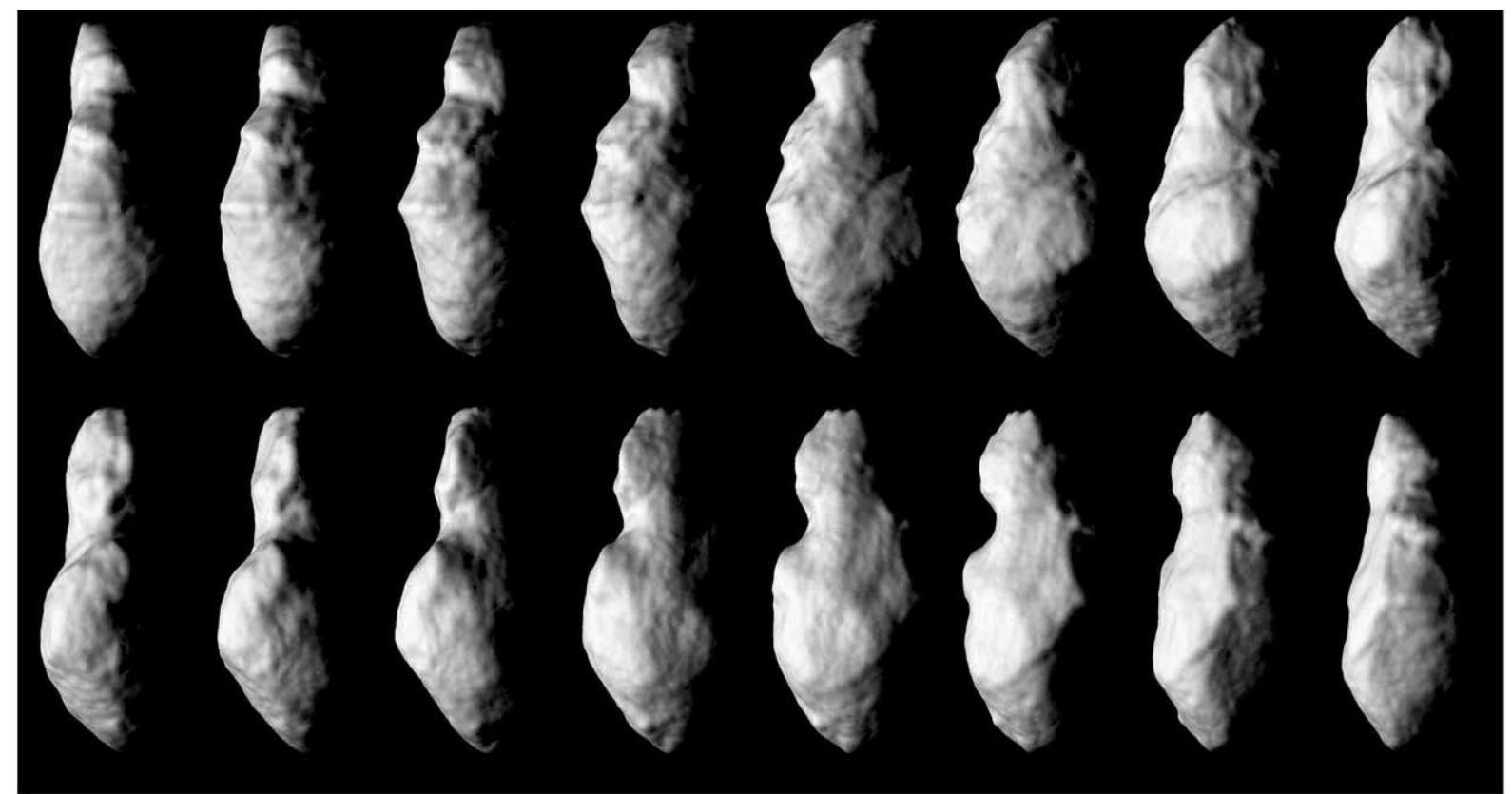

b

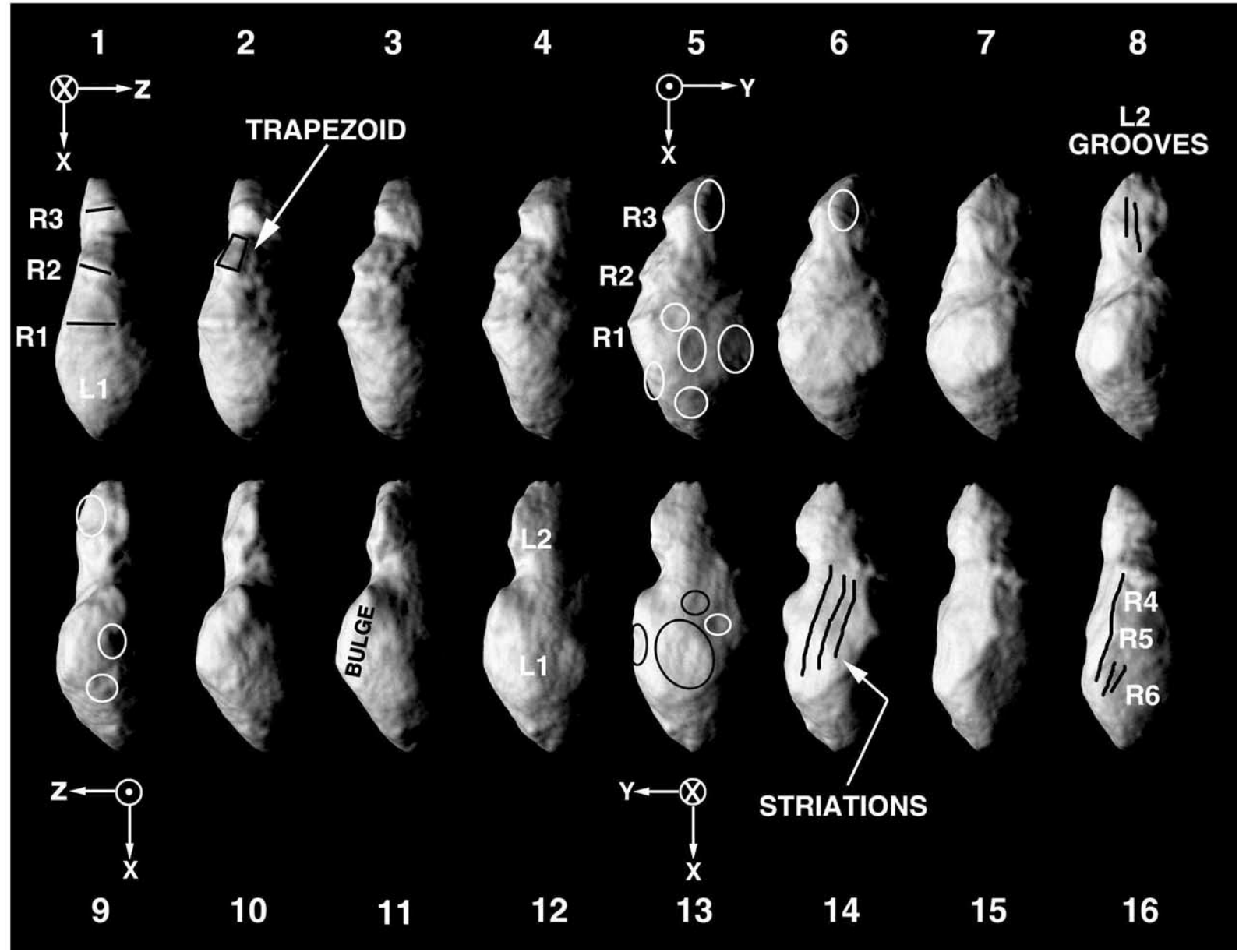

Fig. 4. (a) The Toutatis model rendered at $22.5^{\circ}$ intervals of rotation about the long $(x)$ axis, with illumination coming from the left at a $70^{\circ}$ angle from the viewer's line of sight. In (b), we have indicated features mentioned in the text. 
a

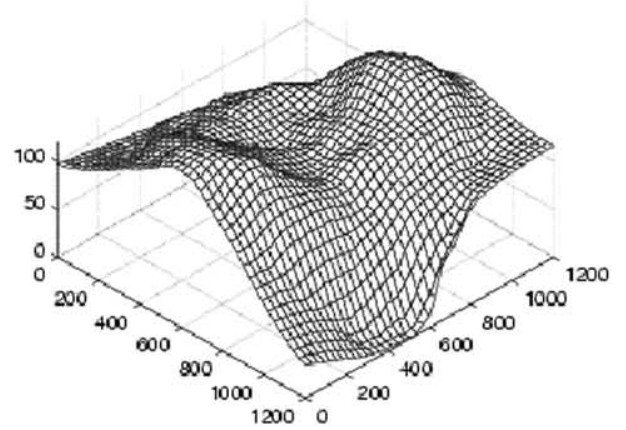

$\mathrm{C} 4$

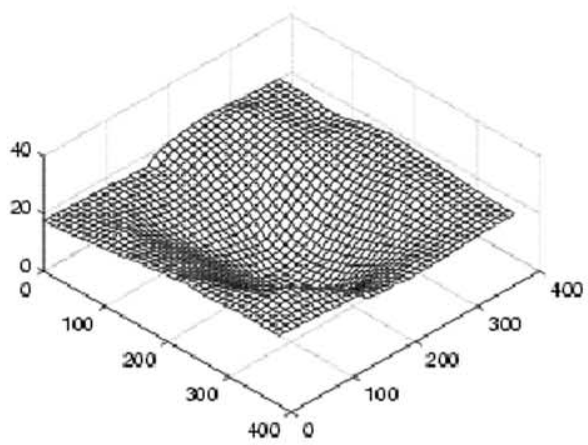

b

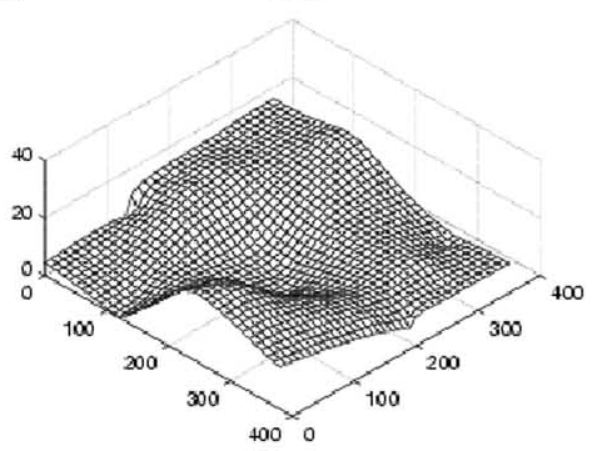

C10

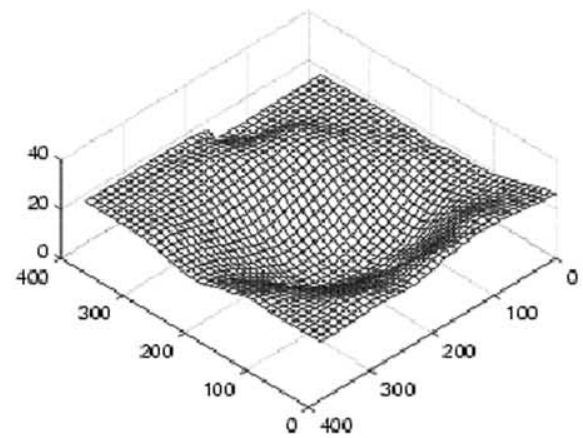

$\mathrm{C} 2$

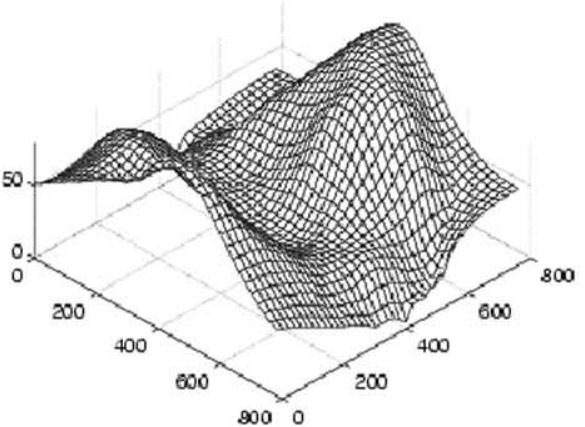

C7

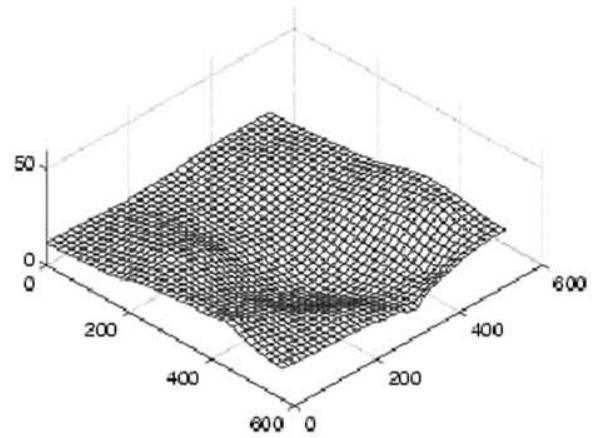

C9

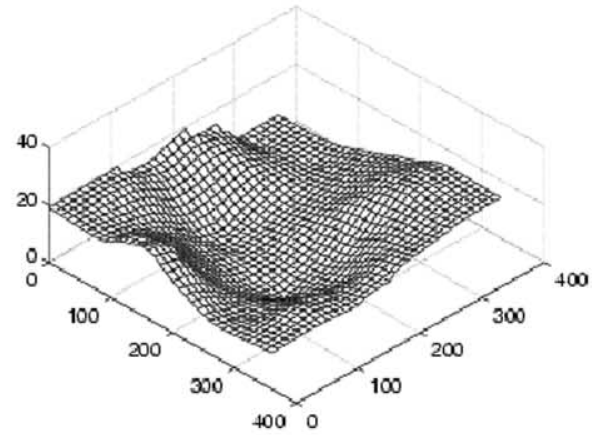

C11

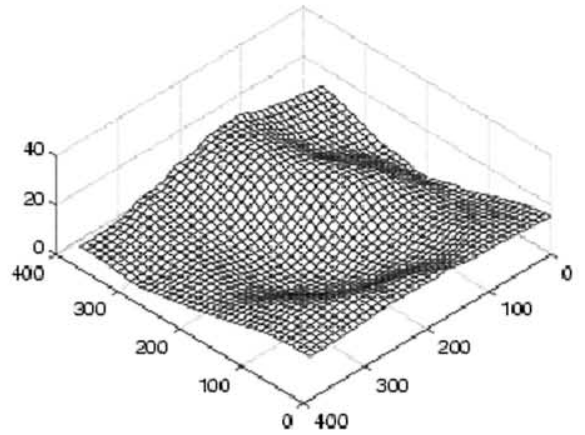

Fig. 5. Topographic relief of eight concavity features. The vertical axis gives elevation (meters) relative to the mean tangent plane. (a) Features $\mathrm{C} 1, \mathrm{C} 2$, $\mathrm{C} 4$, C7; (b) Features C8, C9, C10, C11. The vertical exaggeration is by a factor of 4. 


\section{Shape and topographic features}

Fig. 4 renders the model at $22.5^{\circ}$ intervals of rotation about the long axis, with illumination coming from the left at a $70^{\circ}$ angle from the viewer's line of sight. Fig. $4 \mathrm{~b}$ has been annotated to indicate structural features discussed below.

As noted by HO95, Toutatis' mass distribution along its long axis is not bimodal. Indeed, when viewed along the intermediate-inertia axis (Fig. 3 left frame, right column, or Fig. 4, frame 1 or 9), Toutatis' silhouette looks like a nearly convex, tapered yam. Viewed along the long axis (Fig. 3, left frame, left column), the silhouette is also nearly convex. However, when viewed along the maximum-inertia axis (see Fig. 3, left frame, middle column, or Fig. 4, frame 5 or 13), Toutatis appears bifurcated into a large lobe (L1, about $3 \times 1 \mathrm{~km})$ and a small lobe (L2, about $1 \times 1 \mathrm{~km})$. The lobes are separated by two saddle-like concavities about 400 and $150 \mathrm{~m}$ deep.

\section{Linear features}

HO95 also noted a pattern of three ridges, which they numbered in order of decreasing prominence: $\mathrm{R} 1$ on L1, R2 on L1, and R3 on L2. Each of these ridges is oriented nearly perpendicular to the $y-z$ plane, that is, perpendicular to the long $(x)$ axis. $\mathrm{R} 2$ and $\mathrm{R} 3$ are separated by one of the saddles that define the interlobe neck. $\mathrm{R} 2$ is about $1.0 \mathrm{~km}$ long and is nearly $1 \mathrm{~km}$ from the neck. Indentations connect the ends of R2 with the neck, thus defining a nearly trapezoidal piece of terrain, the Trapezoid, that looks like a section of a block. The $-z$ ends of R1 and R2 are connected by a relatively low ridge $\mathrm{R} 4$ which continues past the end of $\mathrm{R} 1$ for about a kilometer. Not quite parallel to this are a several-hundredmeter low ridge, R5, and a low ridge R6 offset from it (Fig. 4, frame 16). An irregularly shaped bulge dominates L1 opposite the two large ridges. In frames 13-16 we see striations that run much of the length of L1. Two linear structures run along L2, roughly parallel to the asteroid's long dimension, one extending nearly the full length of L2 and the other about one-third as long. Some of the sets of parallel linear features remind us of enechelon ridges seen, for example, on Eros (Veverka et al., 2000; Prockter et al., 2002).

We notice no isolated positive-relief features that resemble boulders. This is not surprising, given that ejecta blocks from cratering events into an object this size are expected to be no larger than a few meters across (Lee et al., 1996), well below our model's resolution.

\section{Concavities}

The model's surface shows numerous concavities that look circular enough for them to be considered candidates for craters, with diameters ranging from $\sim 750 \mathrm{~m}$ down to about a hundred meters. The model has four craters with
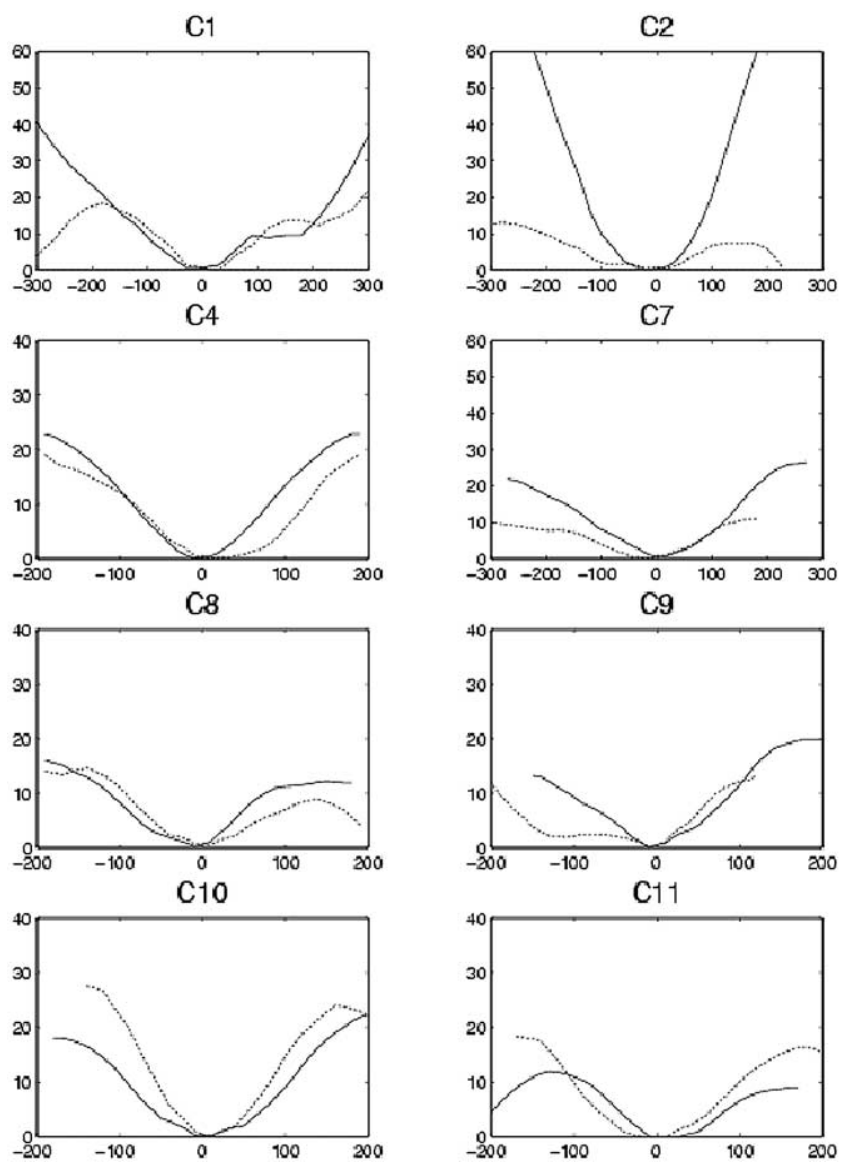

Fig. 6. Topographic profiles through selected concavity features in two orthogonal directions. Dimensions are meters.

diameters of at least $400 \mathrm{~m}$. Given Toutatis' total surface area $\left(22.6 \mathrm{~km}^{2}\right)$, the areal density of craters that large is 0.177 per $\mathrm{km}^{2}$. That statistic plots close to the line fit to crater size-frequency data for Ida (Chapman et al., 1996), which suggests a cratering age of up to 2 byr. There are at least 20 distinct small crater-like concavities in the size range from 100 to $200 \mathrm{~m}$ (e.g., Fig. 4, frames 4, 6, 7, 9, 10); the corresponding lower bound on areal crater density is slightly below the Ida line. However, as noted above, we have suppressed relief in our model solution.

Fig. 5 plots the topographic relief of eight of the concavity features relative to the average tangent plane at that location on the asteroid and Fig. 6 shows profiles cut through those concavities in two orthogonal directions. The depth-to-diameter ratios appear to be within a factor of 2 of 0.1 .

\section{Gravitational slopes}

Fig. 7 shows diverse views of the model with the surface color-coded for gravitational slope ( $S_{\text {grav }}$, the acute angle that a plumb line would make with the local surface normal), as in Fig. 15 of Scheeres et al. (1998). In calculating $S_{\text {grav }}$, uniform density has been assumed. $S_{\text {grav }}$ is lower than 


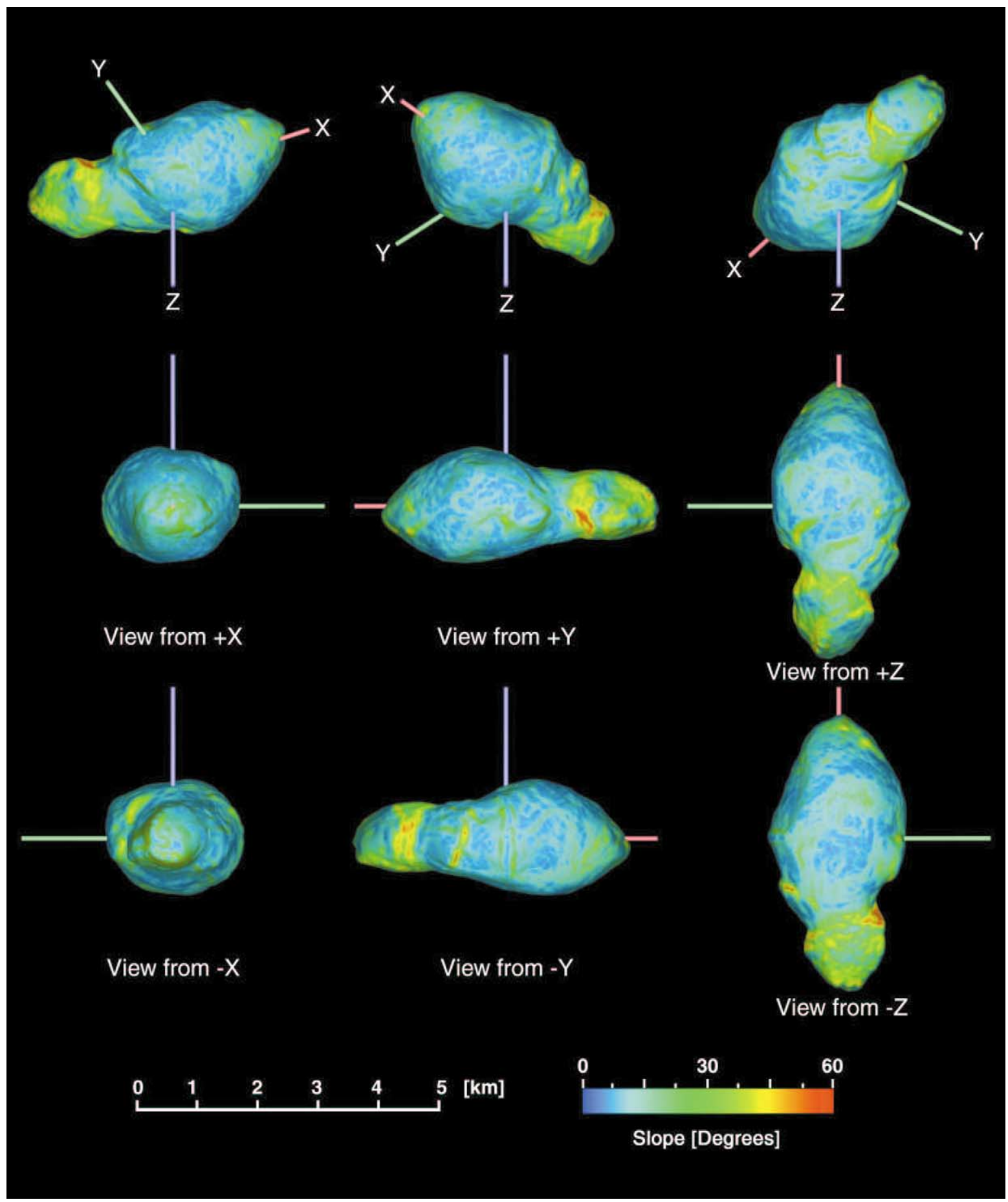

Fig. 7. Gravitational slopes on Toutatis.

$40^{\circ}$ (i.e., the maximum angle of repose for unconsolidated piles of particles) over most of the surface, which therefore may be covered with regolith. However, R2 and R3 have slopes of $40^{\circ}$ and higher (yellow and red areas in the figure, best seen in the rendering from the $-y$ direction), so perhaps those features are exposed bedrock. The smaller lobe, L2, has significantly higher slopes than L1, and part of L2 opposite the ridges has an extended area with slopes larger than $50^{\circ}$ (best seen in the rendering from the $+y$ direction). This region almost surely is exposed bedrock.

\section{Discussion}

The existence of linear and curvilinear ridges and grooves suggests the presence of fractures and some degree 
of internal blockiness at 100-m-to-1-km scales, and therefore a complex history of both compressional and tensional events. Asphaug et al. (1998) demonstrated that the outcome of an impact into a small asteroid is very sensitive to the configuration of pre-existing fractures and voids. That configuration probably was altered repeatedly over Toutatis' collisional lifetime, with each impact altering some fraction of the interior. It may be that neither of the simplistic terms "rubble pile" and "monolith" apply to Toutatis (if, indeed, they apply to any asteroid; see Asphaug et al., 2002, and Richardson et al., 2002). The surface structures evident in our model suggest that the interior contains a geometrically complex arrangement of domains with varying degrees of fracturing, compaction, and tensional coherence. Whether or not Toutatis possesses one or more "pervasive fabrics" like that invoked for Eros is hard to say. On the other hand, the $S_{\text {grav }}$ dichotomy between the asteroid's two lobes suggests structural differences between them, with at least part of L2 being relatively monolithic. We speculate that L1 and L2 are fragments of a precursor body that was disrupted in a subcatastrophic collision, and that the relatively gentle collisional "accumulation" of L1 and L2 to form Toutatis was at least partially responsible for the asteroid's unusual rotation state.

Visual comparison of our Toutatis model with the available spacecraft images of asteroids suggests that relief on Toutatis is at least as severe as that on Eros, Gaspra, Ida, and Dactyl, with a more prominent presence of linear features on Toutatis. An impact that would produce only a several-hundred-meter crater on an object as large as Eros might wreak more havoc on Toutatis, so objects as small as Toutatis might be structurally very different from much larger asteroids.

In penalizing relief in our shape reconstruction, we chose to suppress features rather than to risk showing features that might not be present on Toutatis. Evaluation of whether our degree of caution was warranted will require close-up imaging of Toutatis by a spacecraft.

\section{Acknowledgments}

Work at Washington State University was supported in part by NASA Grant NAGW-4636. Work at the University of Michigan was supported in part by NASA Grant NAG511639. Part of this research was conducted at the Jet Propulsion Laboratory, California Institute of Technology, under contract with the National Aeronautics and Space Administration (NASA). We thank S. Suzuki for constructing the color figure. Critiques by D. Simonelli and an anonymous reviewer significantly improved this paper.

\section{References}

Andrews, A.K., Hudson, R.S., Psaltis, D., 1995. Optical-radar imaging of scale models for studies in asteroid astronomy. Opt. Lett. 20, 23272329 .
Asphaug, E., Ryan, E.V., Zuber, M.T., 2002. Asteroid interiors, in: Bottke, W., Cellino, A., Paolicchi, P., Binzel, R.P. (Eds.), Asteroids III. Univ. of Arizona Press, Tucson, AZ, 463-484.

Asphaug, E., Ostro, S.J., Hudson, R.S., Scheeres, D.J., Benz, W., 1998. Disruption of kilometre-sized asteroids by energetic collisions. Nature 393, 437-440 .

Bard, Y., 1974. Nonlinear Parameter Estimation. Academic Press, New York.

Chapman, C.R., Ryan, E.V., Merline, W.J., Neukum, G., Wagner, R., Thomas, P.C., Veverka, J., Sullivan, R.J., 1996. Cratering on Ida. Icarus $120,77-86$.

Hudson, R.S., Ostro, S.J., 1995. Shape and non-principal axis spin state of Asteroid 4179 Toutatis. Science 270, 84-86.

Hudson, R.S., Ostro, S.J., 1998. Photometric properties of Asteroid 4179 Toutatis from lightcurves and a radar-derived physical model. Icarus 135, 451-457.

Lee, P., Veverka, J., Thomas, P.C., Helfenstein, P., Belton, M.J.S., Chapman, C.R., Greeley, R., Pappalardo, R.T., Sullivan, R., Head, J.W., III, 1996. Ejecta blocks on 243 Ida and on other asteroids. Icarus 120 , 87-105.

Ostro, S.J., Hudson, R.S., Jurgens, R.F., Rosema, K.D., Winkler, R., Howard, D., Rose, R., Slade, M.A., Yeomans, D.K., Giorgini, J.D., Campbell, D.B., Perillat, P., Chandler, J.F., Shapiro, I.I., 1995. Radar images of Asteroid 4179 Toutatis. Science 270, 80-83.

Ostro, S.J., Hudson, R.S., Rosema, K.D., Giorgini, J.D., Jurgens, R.F., Yeomans, D., Chodas, P.W., Winkler, R., Rose, R., Choate, D., Cormier, R.A., Kelley, D., Littlefair, R., Benner, L.A.M., Thomas, M.L., Slade, M.A., 1999. Asteroid 4179 Toutatis: 1996 radar observations. Icarus 137, 122-139.

Prockter, L., Thomas, P., Robinson, M., Joseph, J., Milne, A., Bussey, B., Veverka, J., Cheng, A., 2002. Surface expressions of structural features on Eros. Icarus 155, 75-93.

Richardson, D.C., Leinhardt, Z.M., Melosh, H.J., Bottke Jr., W.F., Asphaug, E., 2002. Gravitational aggregates: evidence and evolution, in: Bottke, W., Cellino, A., Paolicchi, P., Binzel, R.P. (Eds.), Asteroids III. Univ. of Arizona Press, Tucson, AZ, 501-515.

Scheeres, D.J., Ostro, S.J., Hudson, R.S., Suzuki, S., de Jong, E., 1998. Dynamics of orbits close to Asteroid 4179 Toutatis. Icarus 132, 53-79.

Scheeres, D.J., Ostro, S.J., Werner, R.A., Asphaug, E.I., Hudson, R.S., 2000. Effects of gravitational interactions on asteroid spin states. Icarus 147, 106-118.

Spencer, J.R., Akimov, L.A., Angeli, C., Angelini, P., Barucci, M.A., Birch, P., Blanco, C., Buie, M.W., Caruso, A., Chiornij, V.G., Colas, F., Dentchev, P., Dorokhov, N.I., Cristina De Sanctis, M., Dotto, E., Ezhkova, O.B., Fulchignoni, M., Green, S., Harris, A.W., Howell, E.S., Hudecek, T., Kalashnikov, A.V., Kobelev, V.V., Korobova, Z.B., Koshkin, N.I., Kozhevnikov, V.P., Krugly, Y.N., Lazzaro, D., Lecacheux, J., MacConnell, J., Mel'nikov, S.Y., Michalowski, T., Mueller, B.E.A., Nakamura, T., Neese, C., Nolan, M.C., Osborn, W., Pravec, P., Riccioli, D., Shevchenko, V.S., Shevchenko, V.G., Tholen, D.J., Velichko, F.P., Venditti, R., Wisniewski, W., Young, J., Zellner, B., 1995. The lightcurve of 4179 Toutatis: evidence for complex rotation. Icarus 117, 71-89.

Veverka, J., Thomas, P.C., Helfenstein, P., Lee, P., Harch, A., Calvo, S., Chapman, C., Belton, M.J.S., Klaasen, K., Johnson, T.V., Davies, M., 1996. Dactyl: Galileo observations of Ida's satellite. Icarus 120, 200211.

Veverka, J., Robinson, M., Thomas, P., Murchie, S., Bell, J.F., Izenberg, N., Chapman, C., Harch, A., Bell, M., Carcich, B., Cheng, A., Clark, B., Domingue, D., Dunham, D., Farquhar, R., Gaffey, M.J., Hawkins, E., Joseph, J., Kirk, R., Li, H., Lucey, P., Malin, M., Martin, P., McFadden, L., Merline, W.J., Miller, J.K., Owen, W.M., Peterson, C., Prockter, L., Warren, J., Wellnitz, D., Williams, B.G., Yeomans, D.K., 2000. NEAR at Eros: imaging and spectral results. Science 289, 2088 2097. 